\title{
Level of understanding of co-trimoxazole use among HIV infected, recurrent pulmonary tuberculosis suspects at a national referral tuberculosis clinic in Kampala, Uganda: \\ a qualitative analysis.
}

Alphonse Okwera ${ }^{1}$, David K. Mafigiri², David Guwatudde ${ }^{3}$, Christopher Whalen ${ }^{4}$, Moses Joloba ${ }^{5}$

1. Department of Internal Medicine, School of Medicine, College of Health Sciences Makerere University, Kampala

2. Department of Social Work and Social Administration, School of Social Sciences, Makerere University Kampala.

3. Department of Epidemiology and Biostatistics, School of Public Health, College of Health Sciences, Makerere University, Kampala.

4. Department of Epidemiology and Biostatistics, College of Public Health, University of Georgia, Athens, USA.

5. Department of Medical Microbiology, School of Biomedical Sciences, Makerere University Kampala.

\begin{abstract}
Background: Co-trimoxazole use is the standard of care for preventing Pneumocystis jirovecii pneumonia in sub-Saharan Africa but implementation remains slow. Co-trimoxazole is self- administered with uncertain adherence. Knowledge of co-trimoxazole use among HIV infected persons is unknown.

Objectives: To assess knowledge, attitudes and practices of co-trimoxazole use among HIV infected adults evaluated for recurrent PTB in Kampala, Uganda.

Methods: A qualitative study utilizing 5 focus group discussions among $30 \mathrm{HIV}$ infected PTB suspects at the national referral tuberculosis treatment centre in Kampala.

Results: Males and females had similar median ages. $80 \%$ were currently on co-trimoxazole and $50 \%$ of participants were on HAART. Majority of participants defined co-trimoxazole as an analgesic. Few noted co-trimoxazole was a drug to treat cough and chest pain. However, few responses revealed that co-trimoxazole prevents opportunistic diseases among PLHIV. Most of participants believed HAART and anti-TB drugs work as co-trimoxazole thus it should not be taken together with them. This belief may lead to increased risk of opportunistic infections, morbidity and mortality.

Conclusions: We revealed gaps in understanding of co-trimoxazole use among study participants. We therefore recommend that more facts about co-trimoxazle as prophylaxis against P. jirovecii, bacterial and diarrheal pathogens should be incorporated in VCT fact sheets.
\end{abstract}

Key words: Recurrent tuberculosis; HIV; Co-trimoxazole use; Chemoprophylaxis; Adherence

DOI: http://dx.doi.org/10.4314/ahs.v15i1.7

\section{Introduction}

Co-trimoxazole prophylaxis among children, adults and adolescents living with HIV has been the standard of care for many years in high income countries as compared to resource-limited settings ${ }^{1}$. Co-trimoxazole, a fixed dose combination of sulfamethoxazole

\footnotetext{
Correspondence:

Alphonse Okwera,

Department of Internal Medicine,

School of Medicine, College of Health

Sciences, Makerere University,

P.O. Box 663, Kampala.

Tel: 256752437675

Email: A_okwera@mucwru.or.ug

Fax: 256414533631
}

and trimethoprim, is abroad spectrum antimicrobial agent that targets a range of aerobic gram-positive and gram-negative organisms, fungi and protozoa. Providing co-trimoxazole has been part of the standard of care for preventing Pneumocystis jirovecii pneumonia and toxoplasmosis in the sub-Saharan Africa since 2000 but the implementation has been slow ${ }^{2,3}$. Co-trimoxazole prophylaxis has also been found to reduce mortality among patients on HAART' .

Studies in Mulago Hospital revealed that the prevalence of HIV-infection among new tuberculosis cases decreased from $67 \%$ in $1989^{5}$ to $19 \%$ in $2008^{6}$. WHO estimates the burden of TB/HIV co-infection at 19,836 Ugandan adults in 20107. Progressive drop of CD4 T cells below 200 cells $/ \mu \mathrm{L}$ increases the risks for oppor- 
All HIV infected individuals are offered co-trimoxazole prophylaxis and this is in line with the WHO recommendation $^{1,12}$. Routine counseling and testing (RCT) is offered to every patient with confirmed tuberculosis ${ }^{13}$. In 2009, 17131 of the 44335 (54\%) TB cases in Uganda tested HIV positive of whom $86 \%$ were initiated on co-trimoxazole chemoprophylaxis and $22 \%$ were started on HAART ${ }^{14}$. Co- trimoxazole is self-administered nd therefore adherence cannot be ensured. The exten of knowledge, attitudes and practice of HIV infected persons on co-trimoxazole is also unknown. This study was therefore undertaken to investigate co-trimoxazole use among HIV infected adults being evaluated for recurrent PTB in Kampala.

\section{Methods}

Study design

As part of a large study of prevalence of co-morbidity among HIV-infected recurrent PTB suspects ${ }^{15}$, we conducted a qualitative cross sectional evaluation of perceptions about co-trimoxazole use.

\section{Study setting}

This study was conducted at the national referral tuberculosis treatment centre, Mulago National Referral and Teaching Hospital, Kampala. Between 3500 and 4500 tuberculosis patients $(70-75 \%$ new cases and $25-30 \%$ recurrent cases) are treated annually at this TB treatment centre. Current statistics show that $19 \%$ of TB cases treated here are HIV co-infected ${ }^{6}$. New TB cases with HIV co-infection are treated with four antituberculosis drugs: isoniazid, rifampicin, pyrazinamide and ethambutol for two months of intensive phase followed by isoniazid and rifampicin for four months.

\section{Study population and sample size}

Five FGDs each consisting 6 participants $(\mathrm{N}=30)$ were conducted at the tuberculosis treatment center to determine the knowledge, attitudes and practices towards cotrimoxazole use among people with HIV. HIV infected patients who presented with pulmonary symptoms of cough for two weeks or more being evaluated for recurrent tuberculosis were screened for Pneumocystis jirovecii infection. Other eligibility criteria included: (a)
Table 1: Clinical and socio-demographic characteristics of study participants by gender, Mulago

\section{Hospital, Kampala Ugand}

Characteristics

(c) 18 years or older; (d) willingness and consent to un-

Recruitment and data collection procedures

Participants were selected from a list of those enrolled in the study aiming for equal representation by gender. They were contacted by cell phone and the next immediate study participant was chosen if the randomly selected one was unavailable. FGDs were conducted English or Luganda (local dialect), the two most widely spoken languages in the study area. Two study staff conducted each FGD lasting approximately one hour. FGDs were tape recorded and transcribed into MS Word. The domains covered were: participant s knowledge and awareness of co-trimoxazole use, perceptions about who should be given co-trimoxazole, whether HIV patients with or without TB should take co-trimoxazole, relationship between HIV, TB and cotrimoxazole, duration of use, experiences taking co-trimoxazole and its' side effects.

\section{Ethical considerations}

This study was approved by the Makerere University Medical School Ethics and Research Committee and the Uganda National Council for science and Technoloy. Written informed consent was obtained from all sudy participants

\section{Results}

The majority of participants were males $(57 \%)$ and their median ages was similar for both males 39 (IQR37-44) and for females 40 (IQR 32-44) years. There were also no significant differences in education, marital statu and taking co-trimoxazole. Males (88\%) compared to females $(62 \%)$, reported being employed although this difference was not statistically significant $(\mathrm{X} 2$ test $=$ $2.93, \mathrm{p}=0.190)$. Meanwhilemore females $(62 \%) \mathrm{com}-$ pared to males $(42 \%)$ were taking ARVs although this was not statistically significant, $(\mathrm{X} 2$ test $=1.22, \mathrm{p}=$ $0.462)$. Of note however, female participants had a stadistically significant higher median CD4 T-cell counts of 457 (IQR 319-636)/L than males CD4 count of 207 IQR 38-451), $\mathrm{p}=0.021$ (Table 1)

Marital status

married

count/L (IQR)

On ARV

Mean age (years) moxazole noted:

\section{Gender}

Female $n=13(\%)$

$8(62)$

$10(77)$

Employment status
employed
Education
Not beyond primary level
Marital status

Median CD4 T-cell

Taking co-trimoxazole

$207(38-451)$

$7 \quad(41)$

$39(37-44)$

$457(319-636)$
$11(85)$
$8(62)$
$40(32-44)$

$\mathrm{X} 2$ Test

P-value

There were no significant statistical differences in clinical and socio-demographic characteristics by gender except the median CD4 T-cell counts ( $\mathrm{X} 2$ test $=7.03, \mathrm{p}=0.021$ ).

\section{Participant`s knowledge and awareness of co-tri-}

"co-trimoxazole is everywhere in our hospitals and and reported to have used it to treat symptoms like according to the participants. majority described cocough at least once in their life time, as one participant trimoxazole as an analgesic (Table 2).

Table 2: Responses of study participants on their understanding the term co-trimoxazole in Mulago Hospital, Kampala Uganda.

\section{Responses}

It is a pain killer

It is an antibiotic that treats any disease

It is any kind of treatment

A drug for cough and chest pain

A drug that protects us HIV-positive from other diseases Total

$\begin{array}{lc}\text { Number of times } & \text { Percentage } \\ 15 & 51.7 \\ 2 & 6.9 \\ 1 & 3.5 \\ 1 & 3.5 \\ 10 & 34.5 \\ 29 & 100\end{array}$


Some participants reported co- trimoxazole to be medicine given to those with decreased immunity to preven them from getting opportunistic infections associated with HIV/AIDS. The quotes below further illustrates this observation:

"When they found out that I was HIV positive, health workers started me on co-trimoxazole and what they told me was co-trimoxazole is going to help in protecting me from diseases like malaria, diarrhea and cough. So co-trimoxazole is medicine given to those with low immunity like me."

\section{Who should be given co-trimoxazole?}

Majority of participants believed that co-trimoxazole was for treatment of HIV positive patients.

"Co-trimoxazole is always given to HIV positive patients to protect them from all illnesses since their bodies are weak." as one participant noted.

A few thought co-trimoxazole should be taken by anybody regardless of HIV status as one participant no ed:

".... everyone should swallow co-trimoxazole whether HIV positive or not because we are told that co-trimoxazole prevents the body from becoming weak....'

\section{Should HIV patients with or without TB take co-} trimoxazole?

Majority of patients believed co-trimoxazole use by HIV-infected persons is important since it prevent other opportunistic infections. For those participants who were co-infected with both TB and HIV, majority noted that co-trimoxazole should be taken regardless of the disease they had. Notably, some participants thought there is no need to take co-timoxazole because they perceived it and the TB drugs to treat the same diseases. Thus taking co-trimoxazole and anti-TB drugs would be wastage and an increase on the pill burden. One participant believed there is no need to take cotrimoxazole with anti-TB drugs since health workers never instructed him to do so during the period he was on TB treatment although he is HIV infected as expressed below:

"No, he/she should not take it because co-trimoxazole has nothing to do with TB. When I was found to have $\mathrm{TB}$, health workers never instructed me to take co-trimoxazole. So I think this person should only take TB drugs and ARVs only."
Relationship between HIV, TB and co-trimoxazole ondents believed there is a link between and $\mathrm{TB}$, attributing this to the high prevalence of $\mathrm{TB}$ among HIV infected patients as expressed below:

"Before I was HIV positive I used to hear about TB but after becoming HIV positive I developed TB and many of my HIV positive colleagues in the clinic I attend developed TB. This therefore means TB is a common disease amongst us (HIV positive persons)."

Some respondents believed there is no relationship between the two diseases suggesting that TB is a disease which can affect both HIV infected and non-infected persons.

Very many people out there have been diagnosed with TB yet they are not HIV positive so there is no link between TB and HIV because even those who are not HIV positives can also be affected by the disease."

Should an HIV patient on anti-TB treatment also take co-trimoxazole?

Majority of the participants reported that HIV patients on TB treatment could take co- trimoxazole with antiTB drugs attributing this to the perceived preventive activity of co- trimoxazole and reducing the incidence of opportunistic infections among PLHIV. However, some respondents believed even though co-trimoxazole is critical in the decision to prescribe co-trimoxazole to someone as quoted below:

"...it depends on the strength of the body because I was stopped from swallowing co- trimoxazole when was taking TB medicine. The health workers told me my body may not withstand the strength of both TB medicine and co-trimoxazole and I was left on TB medicine until the treatment was over then I resumed my co-trimoxazole.'

Notably, some respondents believed there is no need for HIV-infected patients to continue taking co-trimoxazole along with $\mathrm{TB}$ drugs because they perceived both to contain the same active ingredients for treating chest infections.

Should HIV patients on ARVs also take co-trimoxMajority of the participants thought it was important for a patient to take co-trimoxazole and ARVs simultaneously. Respondents believed that co-trimoxazole would continue protecting the body from other diseases as reported below:

"It is very important for an HIV patient to continue with co-trimoxazole although he/she is swallowing ARVs. For example; I was put on ARVs but I was encouraged by the health workers to continue with co-trimoxazole because co-trimoxazole was to prevent other diseases like diarrhea which may affect my body and am now fine."

Some respondents felt it is not necessary for HIV infected persons to continue with co- trimoxazole. The believed that ARVs are as effective as co-trimoxazole. Thus if an HIV infected person were to continue with the two kinds of medicine, this would be increasing their pill burden.

\section{For how long should one take co-trimoxazole?}

Majority of the participants never knew for how long one should take co-trimoxazole. Some believed co-trimoxazole should be taken as a lifelong treatment While others thought that health care workers should determine the duration.

"I know we are supposed to take co-trimoxazole forever. But I think at one moment my health workers may decide to put me off this medicine especially when $\mathrm{m}$ CD4 cells have gone high because the same thing wa done to my friend and she is now fine," as explained by one participant

On the other hand, some participants believed that someone should stop taking co-trimoxazole if he or she develops side effects particularly when the side effects are severe and can lead to death as expressed below:

"When I was started on co-trimoxazole a few days later I developed a very severe skin rash and burns all over the body and after talking to the health workers, they decided to stop me and instead I was given anothe medicine (dapsone) which I am currently using."

Notably, some participants believed a patient could stop at any time he/she feels better. xperiences with co-trimoxazole

All participants reported improvement in their quality of life after taking co-trimoxazole, as shown by significant reduction in pain and incidences of opportunistic infections. However, some respondents expressed the view that despite perceived improvement in their lives, they still face a challenge of taking co-trimoxazole every day as this is cumbersome.

"Co-trimoxazole has improved our lives, for example I used to feel weak, headache and fevers but currently I am fine. However, this does not mean that it is easy to swallow co- trimoxazole regularly. Imagine every day must swallow two tablets; for sure this makes it very cumbersome."

\section{Side effects of co-trimoxazole}

Majority of the participants had not experienced sideeffects of co-trimoxazole although they heard from their friends. The commonest side effects heard included skin rash, itching of the body and burning sensations in the stomach. Many of respondents, who had not experienced side-effects of co-trimoxazole thus far, expressed their fear of risks of adverse events of cotrimoxazole in future they believed as a consequence of its long term use.

\section{day?}

Most participants believed that it is not good to stop taking co-trimoxazole. They believed that without cotrimoxazole, opportunistic diseases will infect them and this will increase the incidences of morbidity and possibly mortality among them. The quote below illustrates this perception.

"I have seen many of my colleagues, who stopped swallowing co-trimoxazole and developed diseases and later died."

The study established that all participants had ever heard about co-trimoxazole and majority had taken it. Majority demonstrated that co-trimoxazole prevents opportunistic infections among HIV infected individuals with low immunity. They also indicated that HIV and TB were linked, and co-trimoxazole could be used concurrently with anti-tuberculosis medications and HAART Most respondents were also aware of the need for daily use. Similarly, most had ever heard of side effects of 
co-trimoxazole and were aware that treatment had to be discontinued if side effects events were experienced. However there were mixed views about duration of co- trimoxazole use. Some participants believed that co-trimoxazole use was lifelong treatment while others believed health care workers determined the duration. In this study, the significantly low CD4 T-cell count among males may imply that they present to health care facilities late andtherefore more likely to be very sick. Moreover in the main study (15), 8.3\% (8/96) of male had P.jirovecii as compared to $4.9 \%(4 / 82)$ female although this study was not designed to address this aspect of the finding. Further studies in this setting show that males have generally poorer health seeking behavior compared to females ${ }^{24,25}$.

Despite overall wide knowledge, few respondents had important knowledge gaps and misconceptions resulting from real life experiences of co-trimoxazole use. For example, some participants perceived co-trimoxazole as an analgesic. Others believed that co-trimoxazole is exclusively for HIV-infected people. The later belief is quite positive for encouraging the use of $\mathrm{CO}^{-}$ trimoxazole by HIV-infected individuals. The benefit of this is consistent with findings of one study conducted in Uganda and Zimbabwe $e^{4}$. There were divided views among participants whether or not to administer co-trimoxazole to HIV infected patients taking both HAART and anti-tuberculosis drugs. Those participants who support concurrent use of co-trimoxazole believe that co-trimoxazole does reduce the incidents of opportunistic infections among PLHIV. This shared view is encouraging and consistent with $\mathrm{WHO}$ recommendations on use of co-trimoxazole by PLHIV Similarly, several studies have shown that prophylaxis with co- trimoxazole reduced mortality in HIV positive adults with confirmed tuberculosis ${ }^{16-19}$.

Those who disagree with concurrent use of co-trimoxazole by PLHIV on both HAART and anti- tuberculosis therapy argue that anti-tuberculosis drugs work like co-trimoxazole, hence it is inappropriate to take it Anti-tuberculosis drugs including rifampicin, isoniazid, ethambutol, streptomycin and pyrazinamide are used to treat active tuberculosis. This means that these drugs are active mainly against $\mathrm{M}$. tuberculosis microorganisms that cause tuberculosis disease than other opportunistic diseases. Meanwhile co-trimoxazole is used to prevent and treat other opportunistic diseases such s bacterial pneumonia, Pneumocystis jirovecii pneumonia, toxoplasmosis, Isospora beri diarrhea and typhoidal salmonelosis among PLHIV ${ }^{20-23}$. Therefore if these participants stopped taking co-trimoxazole, the risks of infection with opportunistic pathogens, incidence of opportunistic diseases and mortality would increase among them ${ }^{19}$. A well designed information, education and communication (IEC) package needs to be put in place by the $\mathrm{MoH}$ as an intervention strategy to address this misconception and knowledge gaps.

Although majority of participants thought that co-trimoxazole shou on HAART, there was significant misconception that co-trimoxazole should not be taken for long because HAART works like co-trimoxazole. These knowledge gaps would likely contribute to low co-trimoxazole adherence which can lead to increase of opportunistic diseases.

There has been evolving guidelines on when to stop co-trimoxazole prophylaxis. Previous WHO guidelines recommend that co-trimoxazole prophylaxis can be stopped if one is on HAART and CD4 is above 200 cells $/ \mu \mathrm{L}$ for 6 months $^{1}$. Meanwhile the ministry of health $(\mathrm{MoH})$ guideline recommends co-trimoxazole prophylaxis for life in the absence of HAART, but in conclusive for patients on HAART and CD4 count $>200$ cells $/ \mu \mathrm{L}^{13}$. Although some study participants expressed the view that co-trimoxazole prophylaxis has improved their health by reducing the incidences of opportunistic infections, many of them face challenges. One of the biggest challenges is adherence to long term medication. In this study, the following factors were identified as contributing to negative influence on adherence: cumbersomeness to take co- trimoxazole daily, sid effects of co-trimoxazole and advice from relative to discontinue administration of co-trimoxazole Low adherence to co-trimoxazole increases risk of development of resistance by microorganisms. Given the emergence of resistance to trimethoprim-sulfametoxazole among many bacteria ${ }^{26}$, and a more recen study that found co- trimoxazole resistance to Streptococcus pneumonia of $91 \%$ and to non-typhoidal salmonella of $67 \% 0^{27}$ concern has focused on the potential development of resistant Pneumocystis. In the Uganda context, co-trimoxazole is widely available in the country. Studies on use of medicines especially antibiotic use in $\mathrm{Uganda}^{28}$ show that, drugs including antibiotics, are widely available over the counter with or without Acknowledgements prescriptions in pharmacies and most drug shops. We also have drug hawkers within the public commuter taxi parks and bus stations who likewise advise patients on what medication to take including antibiotics ${ }^{29}$. These situations may lead to irrational use of antibiotics including co-trimoxazole. The prescription practices by clinicians in Uganda may also encourage irrational antibiotic use despite the existence of national standard treatment guidelines on rational drug use ${ }^{30,31}$. In terms of administration of co-trimoxazole concurrently with anti-tuberculosis therapy in HIV co-infection with TB, majority of the participants believed that co-trimoxazole should be taken since it prevents other diseases. This particular belief is very positive because it encourages co-trimoxazole use among them.

The study draws strength from use of FGDs because they were helpful in interpreting previously obtained quantitative results and diagnosing the potential problems with a new programmme or service: the number of participants on co-trimoxazole and co-trimoxazole role out programme, in this case.

\section{Limitations}

Limitations of the study include the following: selection of respondents was not stratified by duration of HIV infection, co-trimoxazole and HAART use. And patients with recurrent PTB are likely to have persistent coughs and coughs due to viral pathogens, making it hard to appreciate the role of co-trimoxazole in improving their quality of life.

\section{Conclusion}

There are information gaps in the use of co-trimoxazole among study participants. The belief that HIV infected adults can stop taking co-trimoxazole at any time could lead to low adherence. Females had statistically significantly higher median CD4 T-cell counts than males. For policy and practice, the following recommendations are therefore proposed: as part of a comprehensive HIV Health Care package,all HIV and TB clinics should have IEC materials that address benefits of co-trimoxazole prophylaxis and warning signs of side effects, adherence, concurrent use with other medications like HAART, anti-tuberculosis drugs and drugs that treat other opportunistic infections.
We thank the staff of National Tuberculosis Treatment Centre, Mulago Hospital, Kampala; the study team: Hassard Sempeera, Ochom Emmanuel, Florence Akukuzibwe, Sam Ntambi and Sam Nyole for their support in screening study participants and laboratory aspect of the study and Ismael Nyanzi Ddumba for editorial support. Our sincere gratitude goes to our study participants. This study was supported financially by the AIDS International Training and Research Programme (AITRP) at Case Western Reserve University, Grant No: TW000011, funded by the Fogarty International Centre of the National Institutes of Health. USA.

\section{References}

1. WHO. Guidelines on Co-trimoxazole prophylaxis for HIV-related infections among children, adolescents and adults. Recommendation for a public health approach Geneva: World Health Organization; 2006 Contract No.: Document Number.

2. Mermin J, Lule J, Ekwaru JP, Malamba S, Downing R, Ransom R, et al. Effect of co-trimoxazole prophylaxis on morbidity, mortality, CD4-cell count, and viral load in HIV infection in rural Uganda. Lancet. 2004 Oct 1622;364(9443):1428-34.

3. Watera C, Todd J, Muwonge R, Whitworth J, Nakiyingi-Miiro J, Brink A, et al. Feasibility and effectiveness of cotrimoxazole prophylaxis for HIV-1-infected adults attending an HIV/AIDS clinic in Uganda. J Acquir Immune Defic Syndr. 2006 Jul;42(3):373-8.

4. Walker AS, Ford D, Gilks CF, Munderi P, Ssali F, Reic A, et al. Daily co-trimoxazole prophylaxis in severely immunosuppressed HIV-infected adults in Africa started on combination antiretroviral therapy: an observational analysis of the DART cohort. Lancet. [Research Support, Non-U.S. Gov't]. 2010 Apr10;375(9722):1278-86 5. Eriki PP, Okwera A, Aisu T, Morrissey AB, Ellner JJ, Daniel TM. The influence of human immunodeficiency virus infection on tuberculosis in Kampala, Uganda. Am Rev Respir Dis. 1991Jan;143(1):185-7.

6. Charlebois E, Nanteza-Walusimbi N, Okwera A, Srikantiah P, Murnane P, Morin S, et al. Results of a Randomised Controlled Trial of Home Based Verses TB Clinic Based HIV Voluntary Counseling and Testing for Family and Household Members of TB Evaluation Patients in Uganda XVIII International AIDS Conference, July 18-23, 2010, MOPE0439; 2010 July 18-23, 2010; Viena, Austria. International AIDS Society; 2010 .

African Health Sciences Vol 15 Issue 1, March 2015 
7.WHO. Global tuberculosis control: surveillance, plan- 17. Mwaungulu F, Floyd S, Crampin WC, Kasimba S, ning, financing: WHO report 2011. Geneva,Switzerland: World Health Organization; 2011 Contract No.: Document Number.

8. Phair J, Munoz A, Detels R, Kaslow R, Rinaldo C, Saah A. The risk of Pneumocystis carinii 63 .

pneumonia among men infected with human immunodeficiency virus type 1. Multicenter AIDS Cohor

Study Group. The New England journal of medicine. [Clinical Trial Multicenter Study Research Support, U.S. Gov't, P.H.S.]. 1990 Jan 18;322(3):161-5.

9. Stansell JD, Osmond DH, Charlebois E, LaVange L, Wallace JM, Alexander BV, et al. Predictors of Pneumocystis carinii pneumonia in HIV-infected persons. Pulmonary Complications of HIV Infection Study Group. American journal of respiratory and critical care medicine. [Multicenter Study Research Support, U.S. Gov't, P.H.S.]. 1997 Jan;155(1):60-6.

10. Hirschtick RE, Glassroth J, Jordan MC, Wilcosky TC, Wallace JM, Kvale PA, et al. Bacterial pneumonia in persons infected with the human immu nodeficiency virus. Pulmonary Complications of HIV Infection Study Group. The New England journal of medicine. [Multicenter Study Research Support, U.S. Gov't, P.H.S.]. 1995 Sep 28;333(13):845-51.

11. Afessa B, Green B. Bacterial pneumonia in hospitalized patients with HIV infection: the Pulmonary Complications, ICU Support, and Prognostic Factors of Hospitalized Patients with HIV (PIP) Study. Chest. [Comparative Study]. 2000 Apr;117(4):1017-22.

12. MoH. National antiretroviral treatment guidelines for adults,adolescents and children. Kampala: Ministry of Health; 2009 June 2009 Contract No.: Documen Number.

13. MoH. Manual of the National Tuberculosis and Leprosy Programme (NTLP). Kampala: Ministry of Health; 2010 Contract No.: Document Number.

14. WHO. Global tuberculosis control: surveillance, planning, financing: WHO Report 2010. Geneva, Switzerland: World Health Organization; 2010 Contract No.: Document Number.

15. Okwera A, Bwanga F, Najjingo I, Mulumba Y, Mafigiri DK, Whalen CC, et al. Aetiology of pulmonary symptoms in HIV-infected smear negative recurrent PTB suspects in Kampala, Uganda: a crosssectional study. PLoS One. 2013;8(12):e82257.

16. Zachariah R, Spielmann M, Chinji C, Gomani P, Arendt V, Hargreaves J. Voluntary counseling, HIV testing and adjunctive co-trimoxazole reduces mortality in tuberculosis patients in Thyolo, Malawi. AIDS.2003;17:1053-61. mortality in human immunodeficiency virus - positive tuberculosis patients in Karonga Disrrict, Malawi. Bull World Health Organ 2004;82:354 63.

18. Nunn J, Mwaba P, Chintu C, Mwinga A, Derbyhire J, Jumla A. Role of co-trimoxazole prophylaxis in reducing mortality in HIV infected adults being treated for tuberculosis: randomized clinical trial. $B M$ J 2008;337:2257.

9. Grimwake K, Sturm A, Nunn J, Mbatha D, Zungu D, Gilks C. Effectiveness of co-trimoxazole prophylaxis on mortality in adults with tuberculosis in rural South Africa. AIDS. 2005;19:163-8.

20. Jones JL, Hanson DL, Dworkin MS, Alderton DL, Fleming PL, Kaplan JE, et al. Surveillance for AIDSdefining opportunistic illnesses, 1992-1997. MMWR CDC surveillance summaries : Morbidity and mortality weekly report CDC surveillance summaries / Centers for Disease Control. 1999 Apr 16;48(2):1-22.

21. Carr A, Tindall B, Brew BJ, Marriott DJ, Harkness L, Penny R, et al. Low-dose trimethoprim- sulfamethoxazole prophylaxis for toxoplasmic encephalitis in patients with AIDS. Annals of internal medicine. [Comparative Study Research Support, Non-U.S. Gov't]. 1992 ul 15;117(2):10611.

22. Anglaret X, Chene G, Attia A, Toure S, Lafont S, Combe P, et al. Early chemoprophylaxis with trimethoprim-sulphamethoxazole for HIV-1-infected adults in Abidjan, Cote d'Ivoire: a randomised trial. Cotrimo-CI Study Group. Lancet. [Clinical Trial Randomized Controlled Trial Research Support, Non-U.S. Gov't]. 1999 May 1;353(9163):1463-8.

23. Wiktor SZ, Sassan-Morokro M, Grant AD, Abouya L, Karon JM, Maurice C, et al. Efficacy of trimethoprimsulphamethoxazole prophylaxis to decrease morbidity and mortality in HIV-1-infected patients with tuberculosis in Abidjan, Cote d'Ivoire: a randomised controlled trial. Lancet. [Clinical Trial Randomized Controlled Trial Research Support, Non-U.S. Gov't Research Support, U.S. Gov't, P.H.S.]. 1999 May 1;353(9163):1469-75.

24. Government of Uganda. Uganda Demographic and Health Survey 2011. Kampala: Uganda Bureau of Statistics, Measure DHS; 2012 Contract No.: Document Number.

25.Uganda Ministry of Health, ICF International. 2011 Uganda AIDS Indicator Survey: Key Findings. Calverton, Maryland, USA: MOH and ICF International; 2012 Contract No.: Document Number.

26. Huovinen P. Resistance to trimethoprim-sufamrthoxazole. Clin Infect Dis. 2001;32:1608-14.
27. Mayanja BN, Todd J, Hughes P, Van der Paal L, Mu- by the rational pharmaceutical management project. gisha JO, Atuhumuza E, et al. Septicaemia in a popula- Arlington, VA: Management Sciences for Health; 2000 tion-based HIV clinical cohort in rural Uganda, 1996- [updated 2000; cited 2011 September, 19]; Available 2007: incidence, aetiology, antimicrobial drug resistance from: http://pdf.usaid.gov/pdf_docs/PNACM445. and impact of antiretroviral therapy. Tropical medicine pdf.

\& international health : TM \& IH. [Research Support, 30. Kafuko JM, Zirambuzaale C, Bagenda D. Ratinal Non-U.S. Gov't]. 2010 Jun;15(6):697-705.

rug use in rural health units of Uganda: Effect of naMusigunzi N, Kamulegeya A. Antibiotic knowledge use. Kampala: Ministry of Health; 2008 Contract No: and behavior at a Ugandan University. Int J Infec Conge 2011; $7:$ :i4

use. Kampala: Ministry of Health; 2008 Contract No:
Document Number. 31. Kamulegeya A, Buwembo W, Rwenyonyi CM. 29. Cabra M, Kisalu A, Hazemba O. Uganda Assess- Knowledge and Antibiotic Prescription Pattern among ment: Drug management for childhood illness. Pub- Ugandan Oral Health Care Providers: A Crosslished for the US Agency for International Development sectional Survey. J Dent Res Dent Clin Dent Prospects 2011;5(3):61-6. 